\title{
A phase 1 study of the safety, tolerability, pharmacokinetics, and pharmacodynamics of TAK-063, a selective PDE10A inhibitor
}

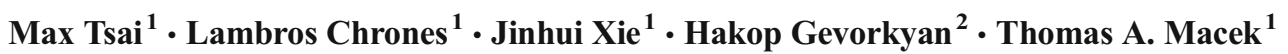

Received: 23 March 2016 / Accepted: 11 August 2016 / Published online: 30 August 2016

(C) The Author(s) 2016. This article is published with open access at Springerlink.com

\begin{abstract}
Rationale Schizophrenia is a complex neuropsychiatric disorder characterized, in part, by impaired dopamine signaling. TAK-063 is a selective inhibitor of phosphodiesterase 10A, a key regulator of intracellular signaling pathways that is highly expressed in the striatum.

Objective Safety, tolerability, and pharmacokinetics of TAK063 were evaluated in a phase 1 study.

Methods Healthy Japanese and non-Japanese volunteers were randomized into dose cohorts of 3,10,30,100,300, and $1000 \mathrm{mg}$. Each fasting volunteer randomly received a single dose of TAK-063 or placebo. Individuals from the 100-mg cohort also received a post-washout, 100-mg dose under fed conditions. A total of 84 volunteers enrolled (14 per cohort). Results The most common drug-related adverse events (AEs) were somnolence $(33.3 \%)$, orthostatic tachycardia (19.7\%), and orthostatic hypotension $(9.1 \%)$. The three severe AEs recorded occurred at the highest doses: orthostatic hypotension $(n=1 ; 300 \mathrm{mg})$ and somnolence $(n=2 ; 1000 \mathrm{mg})$. There were no deaths, serious AEs, or discontinuations due to AEs. TAK-063 exposure increased in a dose-dependent manner. Median $T_{\max }$ was reached 3 to $4 \mathrm{~h}$ postdose. Fed conditions slowed absorption $\left(T_{\max }=6 \mathrm{~h}\right)$ and increased oral bioavailability. Renal elimination was negligible. Safety and pharmacokinetic parameters were similar between Japanese and non-Japanese
\end{abstract}

Electronic supplementary material The online version of this article (doi:10.1007/s00213-016-4412-9) contains supplementary material, which is available to authorized users.

Thomas A. Macek

tom.macek@takeda.com

1 Takeda Development Center Americas, Inc., One Takeda Parkway, Deerfield, IL 60015, USA

2 California Clinical Trials Medical Group, Glendale, CA, USA subjects. Impairments in cognitive function consistent with the effects of other sedative or hypnotic agents were detected using a validated, computerized cognition battery, CNS Vital Signs.

Conclusions TAK-063 was safe and well tolerated at doses up to $1000 \mathrm{mg}$ and demonstrated a pharmacokinetic profile supporting once-daily dosing. Further evaluation of the clinical safety and efficacy of TAK-063 is warranted.

Keywords Schizophrenia $\cdot$ Pharmacokinetics $\cdot$ Safety Phosphodiesterase 10A $\cdot$ Single-rising dose $\cdot$ Oral

\section{Introduction}

Schizophrenia is a neuropsychiatric disorder of complex etiology. Associated with lifelong disability and a poor quality of life, schizophrenia presents as a triad of positive, negative, and cognitive symptoms (Citrome 2014; de Araujo et al. 2012).

Current standard of care (first- and second-generation antipsychotics) are thought to achieve their therapeutic effects primarily through antagonism of the dopamine $\mathrm{D}_{2}$ receptor subtype (Farde et al. 1992; Ginovart and Kapur 2012). Numerous studies have demonstrated the efficacy of antipsychotics in ameliorating positive symptoms (Lieberman et al. 2005), though a significant proportion of patients experience residual symptoms that are not responsive to therapy (Lehman et al. 2004; Tarrier 1987). Further, these drugs have little to modest clinical effects on cognitive and negative symptoms (Citrome 2014; Lehman et al. 2004; Leucht et al. 2009).

Current antipsychotics are often associated with a multitude of adverse events (AEs), including extrapyramidal symptoms (EPS) and hyperprolactinemia, which are due, at least in part, to excessive dopamine $\mathrm{D}_{2}$ receptor antagonism in the striatum and the pituitary gland, respectively (Farde et al. 1992; Ginovart and Kapur 2012). Metabolic disturbances 
affecting weight, lipid profile, and blood glucose levels have also been observed following treatment with current agents or therapies (Ginovart and Kapur 2012; Leucht et al. 2009; Newcomer 2007). These dose-limiting side effects, along with the persistent cognitive, negative, and psychotic symptoms also associated with typical and atypical antipsychotics, underscore the need for new pharmacologic treatment options (Citrome 2014).

TAK-063 is a potent and selective phosphodiesterase 10A (PDE10A) inhibitor in clinical development for the treatment of schizophrenia (Suzuki et al. 2015). PDE10A is an intracellular enzyme that degrades cyclic adenosine monophosphate pathway (cAMP) and cyclic guanosine monophosphate pathway (cGMP)—second messenger molecules with important effects on neuronal excitability (Fujishige et al. 1999; Song et al. 2006; Threlfell et al. 2009). Unlike other phosphodiesterases, PDE10A is selectively expressed in the GABAergic medium-spiny neurons (MSNs), the predominant neuronal population of the striatum (Kreitzer 2009; Sano et al. 2008; Seeger et al. 2003; Suzuki et al. 2015).

In MSNs of both the direct and indirect pathways, PDE10A mediates signaling activity downstream of dopamine $D_{1}$ (direct) and $D_{2}$ (indirect) receptor activation (Sano et al. 2008; Siuciak et al. 2006; Suzuki et al. 2015). PDE10A-mediated modulation of signaling through these two distinct dopamine receptor subtypes affects the sensitivity of MSNs to glutamatergic inputs, and thereby partly regulates the opposing effects of both the direct and indirect pathways (Siuciak et al. 2006; Surmeier et al. 2007). PDE10A may represent an important regulatory locus within the striatal signaling network; it receives inputs from cortical, thalamic, and subcortical areas that regulate sensory processing, motor planning, and goal-directed behavior (Alexander et al. 1986; Macpherson et al. 2014; Perez-Costas et al. 2010). Notably, the clinical expression of schizophrenia includes significant deficits in these and related higher-order processes, making it difficult for patients to perform basic tasks (Citrome 2014; de Araujo et al. 2012).

Taken together, the preclinical data validate PDE10A as a potential target for therapeutic intervention (Grauer et al. 2009; Kehler and Nielsen 2011), and support further investigation of the safety, tolerability, and potential multidimensional efficacy of selective PDE10A inhibitors in people with schizophrenia (Heckman et al. 2016). Here, the results of a phase 1, randomized, placebo-controlled, single-rising dose study exploring the pharmacokinetic (PK), and pharmacodynamic (PD) profiles of TAK-063 in healthy subjects are reported. As prior reports have noted ethnic differences in pharmacokinetics of several compounds, results for Japanese and non-Japanese subjects are reported (Fukunaga et al. 2011).

\section{Methods}

\section{Study design and subjects}

The study was conducted at a single site in the USA in compliance with Institutional Review Board regulations, Good Clinical Practice regulations and guidelines, and all local regulations.

Healthy Japanese and non-Japanese subjects between 18 and 55 years of age were recruited to participate in the study. Criteria for exclusion included prior treatment with any investigational compound within 30 days of the first TAK-063 dose, a positive test for drugs of abuse or nicotine, and an increased risk for suicide. Subjects were randomized into 1 of 6 dose cohorts $(3,10,30,100,300$, and $1000 \mathrm{mg})$, with each cohort containing 11 TAK-063 subjects (five Japanese, six non-Japanese) and three placebo subjects (one Japanese, two non-Japanese) receiving a single dose under fasting conditions. Following a washout period of 7 days, subjects in cohort $3(100 \mathrm{mg})$ received the same dose $(100 \mathrm{mg})$ but under fed conditions.

\section{Study endpoints}

The study assessed safety and tolerability in all subjects for the 4-day study duration. The primary endpoints were the percentage of subjects who experienced at least one treatmentemergent adverse event (TEAE), as well as the percentage of subjects who met predefined criteria for safety laboratory tests, vital sign measurements, and safety parameters following TAK-063 administration. The secondary endpoints were PK parameters for TAK-063 and M-I, assessing drug exposure at each dose, and the effect of food coadministration on TAK-063 PK. Exploratory endpoints included the effects of TAK-063 on domains of cognition as assessed by a computerized battery, CNS Vital Signs (CNSVS).

\section{Safety assessments}

Adverse events, clinical laboratory tests, vital signs, 12-lead electrocardiograms (ECGs), and Columbia-Suicide Severity Rating Scale (C-SSRS) were all monitored. Intensity of AEs was classified as mild, moderate, or severe, and each AE was classified as to its relation to study drug.

Laboratory tests evaluated hematology, serum chemistry, urine, and hormone levels. Vital signs and ECGs were collected at screening, check-in, within $1 \mathrm{~h}$ predose, serially postdose, and at study exit, while the C-SSRS was administered at screening, check-in, and study exit. Any events reported on the C-SSRS and clinically significant laboratory abnormalities were reported as AEs. 


\section{Pharmacokinetic and pharmacodynamic assessments}

Serial plasma and urine samples were collected from all subjects prior to dosing and at specific time points or intervals up to $96 \mathrm{~h}$ postdose. Pharmacokinetic samples were processed immediately and frozen at $-20{ }^{\circ} \mathrm{C}$ as duplicate sets. Plasma concentrations of TAK-063 and its metabolite M-I were subsequently measured by validated liquid chromatographytandem mass spectrometry with a validated range of 0.5 to $1000 \mathrm{ng} / \mathrm{mL}$ for both analytes.

The PK parameters analyzed for TAK-063 and its metabolite $\mathrm{M}-\mathrm{I}$ in plasma and urine included area under the plasma concentration-time curve from time 0 to infinity $\left(A U C_{(0-\text { inf })}\right)$, maximum observed plasma concentration $\left(C_{\max }\right)$, time to reach $C_{\max }\left(T_{\max }\right)$, terminal elimination half-life $\left(T_{1 / 2}\right)$, oral clearance $(\mathrm{CL} / \mathrm{F})$, volume of distribution $\left(\mathrm{V}_{\mathrm{z}} / \mathrm{F}\right)$, and renal clearance $\left(\mathrm{CL}_{\mathrm{r}}\right)$. Metabolite-to-parent ratios were estimated from $C_{\max }$ and $A U C_{(0 \text {-inf })}$ data.

Cognitive flexibility, attention, executive function, memory, reasoning, motor control, psychomotor speed, reaction time, and information processing speed were assessed predose and at 2 and $6 \mathrm{~h}$ following TAK-063 treatment using the CNSVS computerized cognition battery. The CNSVS cognition battery is a well-validated, sensitive measure and included the following tests: verbal and visual memory (VBM and VIM) tests, finger-tapping test, symbol digit coding test, Stroop test, shifting attention test, the nonverbal reasoning test, and the 4-part continuous performance test (Gualtieri and Johnson 2006). Standardized domain scores for cognitive flexibility, composite memory, executive functioning, processing speed, psychomotor speed, reaction time, reasoning, sustained attention, verbal memory, visual memory, and working memory were reported. In all domains, a decrease in the standardized domain score is indicative of impairment.

\section{Statistical analysis}

All statistical analyses were generated using SAS Version 9.2 (SAS, Cary, NC, USA). The PK parameters were derived using non-compartmental methods with WinNonlin Enterprise Version 6.3 (Pharsight Corp., Mountain View, CA, USA). Frequency or descriptive statistics were used for summary of data. Where indicated, percent coefficient of variation was included in the summary of continuous data. All statistical tests were 2-tailed at $\alpha=0.05$ level for significance unless otherwise stated. A power model was used to evaluate dose proportionality by race for fasted subjects; in the event of no observed race effect, dose proportionality was assessed for all subjects. To evaluate the food effect, a paired $t$ test was performed on $T_{\max }$ and natural log-transformed $C_{\max }$, and AUCs. An ANCOVA model with baseline scores and race as covariates was used to assess the effects of TAK-063 on CNSVS domain scores.

\section{Results}

\section{Demographics}

A total of 84 subjects were enrolled in this study, with comparable distribution of male and female subjects in each dose group (Table 1). The mean age and body mass index were similar among all groups and ranged from 30.5 to 36.1 years and 22.6 to $24.5 \mathrm{~kg} / \mathrm{m}^{2}$, respectively. Over $90 \%$ of subjects were of non-Hispanic/non-Latino ethnicity, and the racial distribution across subjects was approximately half Asian, one-quarter Caucasian, and one-quarter Black/African American.

\section{Safety}

No deaths or other serious AEs were reported, and no subject discontinued treatment because of AEs. Across all cohorts, 32 of 66 subjects experienced a total of 57 drug-related AEs (Table 2). The most common drug-related AEs following TAK-063 treatment were somnolence (33.3\%), orthostatic tachycardia (19.7\%), and orthostatic hypotension (10.6\%). Reports of EPS or EPS-like events were infrequent across all subjects - one Japanese subject reported muscle tightness in the 30-mg treatment group. The majority of AEs (55/63; $87.3 \%$ ) were of mild intensity. Three adverse events were reported as severe intensity: one subject had orthostatic hypotension $(300 \mathrm{mg})$, and two had somnolence $(1000 \mathrm{mg})$. The total incidence of TEAEs within Japanese subjects treated with TAK-063 was $43.3 \%$ compared to $52.8 \%$ in nonJapanese subjects (Online Resources 1 and 2). None of the physical examination parameters, clinical laboratory tests, CSSRS findings, or ECGs was considered clinically significant for any subject. There was an increased incidence of abnormal readings for blood pressure and pulse rate, mostly occurring in subjects in the 1000-mg TAK-063 treatment group.

\section{Pharmacokinetic properties of TAK-063}

The mean concentration-time profiles of TAK-063 and M-I in all subjects were measured over a period of $96 \mathrm{~h}$ following administration of single oral doses (Fig. 1). Under fasting conditions, TAK-063 was absorbed with a median $T_{\max }$ value of 3 to $4 \mathrm{~h}$ postdose and eliminated with a mean $T_{1 / 2}$ value of 15 to $25 \mathrm{~h}$ postdose across all subjects (Fig. 1). M-I exhibited a similar mean plasma concentration-time profile to the parent TAK-063. No substantial differences for either analyte were observed between Japanese and non-Japanese subjects (Online Resource 5).

The plasma and urine PK parameters of TAK-063 and M-I under fasting conditions are presented in Table 3. Exposure to TAK-063 was less than dose-proportional with AUC values increasing up to 26 -fold in response to the 333 -fold increase in 


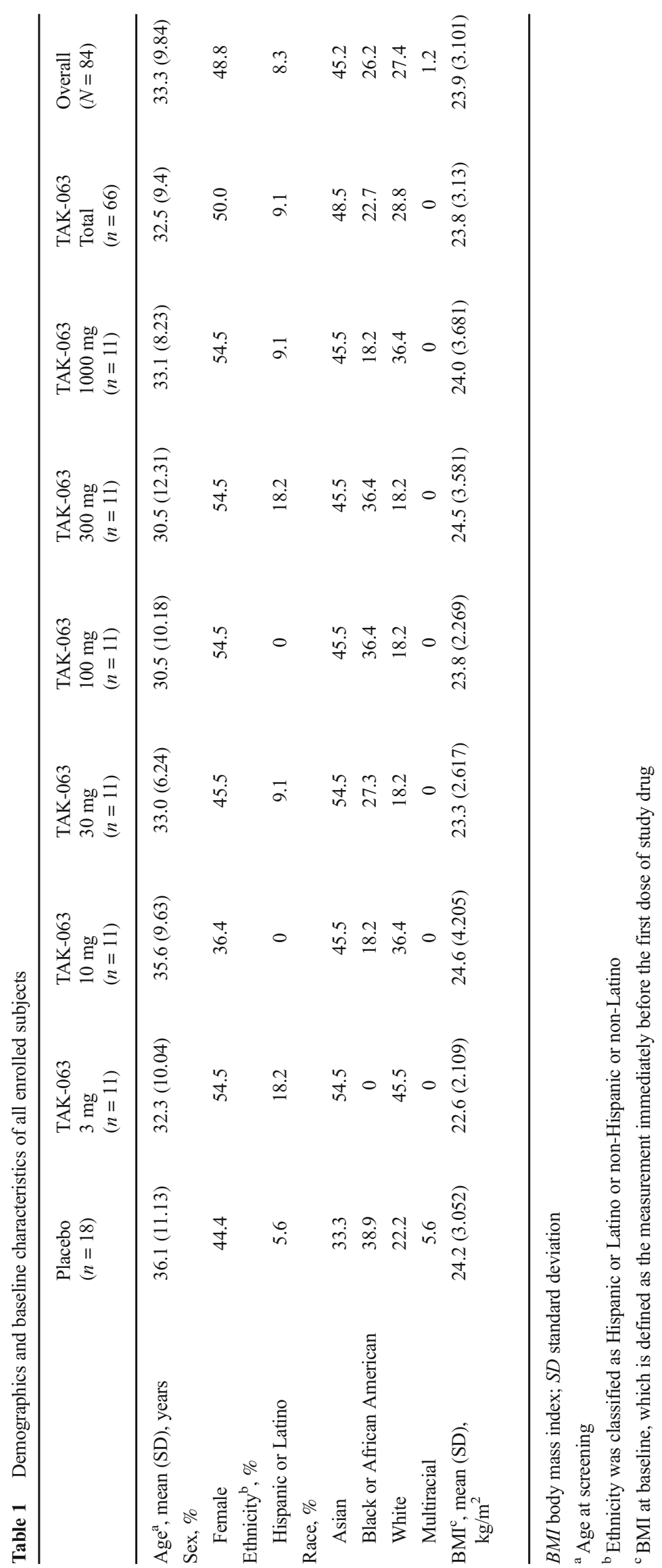


Table 2 Treatment-emergent adverse events across all subjects by dosing group

\begin{tabular}{|c|c|c|c|c|c|c|c|c|}
\hline $\begin{array}{l}\text { Adverse event, } \\
n(\%)\end{array}$ & $\begin{array}{l}\text { Placebo } \\
(n=18)\end{array}$ & $\begin{array}{l}\text { TAK-063 } \\
3 \mathrm{mg} \\
(n=11)\end{array}$ & $\begin{array}{l}\text { TAK-063 } \\
10 \mathrm{mg} \\
(n=11)\end{array}$ & $\begin{array}{l}\text { TAK-063 } \\
30 \mathrm{mg} \\
(n=11)\end{array}$ & $\begin{array}{l}\text { TAK-063 } \\
100 \mathrm{mg} \\
(n=11)\end{array}$ & $\begin{array}{l}\text { TAK-063 } \\
300 \mathrm{mg} \\
(n=11)\end{array}$ & $\begin{array}{l}\text { TAK-063 } \\
1000 \mathrm{mg} \\
(n=11)\end{array}$ & $\begin{array}{l}\text { TAK-063 } \\
\text { Total } \\
(n=66)\end{array}$ \\
\hline Somnolence & 0 & 0 & 0 & $4(36.4)$ & 7 (63.6) & $5(45.5)$ & $6(54.5)$ & $22(33.3)$ \\
\hline $\begin{array}{l}\text { Orthostatic } \\
\text { tachycardia }\end{array}$ & $1(5.6)$ & $2(18.2)$ & 0 & $1(9.1)$ & $2(18.2)$ & $1(9.1)$ & 7 (63.6) & $13(19.7)$ \\
\hline $\begin{array}{l}\text { Orthostatic } \\
\text { hypotension }\end{array}$ & $3(16.7)$ & 0 & 0 & 0 & $1(9.1)$ & $1(9.1)$ & $5(45.5)$ & $7(10.6)$ \\
\hline Vomiting & 0 & 0 & 0 & 0 & $1(9.1)$ & $1(9.1)$ & $1(9.1)$ & $3(4.5)$ \\
\hline Nausea & 0 & 0 & 0 & $2(18.2)$ & 0 & 0 & $1(9.1)$ & $3(4.5)$ \\
\hline Dizziness & 0 & 0 & 0 & $1(9.1)$ & 0 & $1(9.1)$ & $1(9.1)$ & $3(4.5)$ \\
\hline Dysarthria & 0 & 0 & 0 & 0 & 0 & 0 & $1(9.1)$ & $1(1.5)$ \\
\hline Headache & $2(11.1)$ & 0 & 0 & 0 & $1(9.1)$ & $1(9.1)$ & 0 & $2(3.0)$ \\
\hline Anxiety & 0 & 0 & 0 & 0 & 0 & 0 & $1(9.1)$ & $1(1.5)$ \\
\hline Hypotension & 0 & 0 & 0 & 0 & $1(9.1)$ & 0 & 0 & $1(1.5)$ \\
\hline Blurred vision & 0 & 0 & 0 & 0 & 0 & 0 & $1(9.1)$ & $1(1.5)$ \\
\hline Epistaxis & 0 & 0 & 0 & $1(9.1)$ & 0 & 0 & 0 & $1(1.5)$ \\
\hline $\begin{array}{l}\text { Muscle } \\
\text { tightness }\end{array}$ & 0 & 0 & 0 & $1(9.1)$ & 0 & 0 & 0 & $1(1.5)$ \\
\hline
\end{tabular}

TAK-063 dose investigated in this study (Fig. 2). The intersubject variability of TAK-063 $C_{\max }$ and $A U C_{(0 \text {-inf })}$ values was generally moderate across doses (18-81\% coefficient of variation). Clearance of TAK-063 (CL/F) increased with dose whereas renal elimination was negligible (Table 3). Similar PK properties were observed for TAK-063 M-I (M-I to parent $C_{\max }$ and AUC ratios: $0.82-0.97$ and $0.78-1.04$ ). PK parameters for Japanese and non-Japanese subjects are reported in Online Resources 3 and 4. For both TAK-063 and M-I, no substantial differences in pharmacokinetics were observed between Japanese and non-Japanese subjects.

An evaluation of the effects of food on absorption and oral bioavailability of TAK-063 following a 100-mg dose found that TAK-063 was absorbed more slowly when coadministered with a standard meal, (median $T_{\max }=6 \mathrm{~h}$, compared with 3 to $4 \mathrm{~h}$ under fasting condition), and oral bioavailability of TAK-063 was approximately 2-fold greater than under fasting conditions (Table 4). Terminal elimination, fraction of dose excreted, and renal clearance were not substantially altered under fed conditions. M-I showed similar PK properties to TAK-063 in the fasting and fed arms, with M-I to parent ratios of 0.97 and 1.00; 0.99 and 0.98 ; and 1.04 and 1.00 for $C_{\max }, A U C_{(0-\text { tlqc })}$, and $A U C_{(0-\text { inf })}$, respectively. No observed differences were apparent between Japanese and non-Japanese subjects in either fasting or fed condition (data not shown).

\section{Effects of TAK-063 on cognition}

An overall dose effect of TAK-063 relative to placebo was observed at 2 and $6 \mathrm{~h}$ postdose (Online Resource 6) on the domains of cognitive flexibility, executive functioning,
Fig. 1 Mean plasma concentration-time profiles of TAK-063 and M-I (metabolite) across all subjects by dosing group
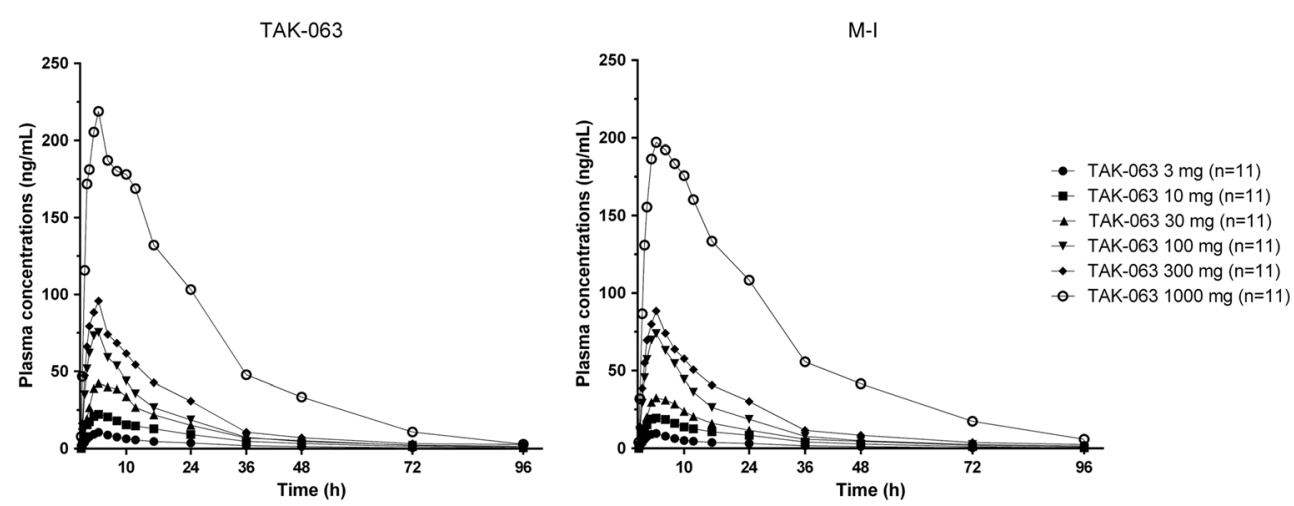


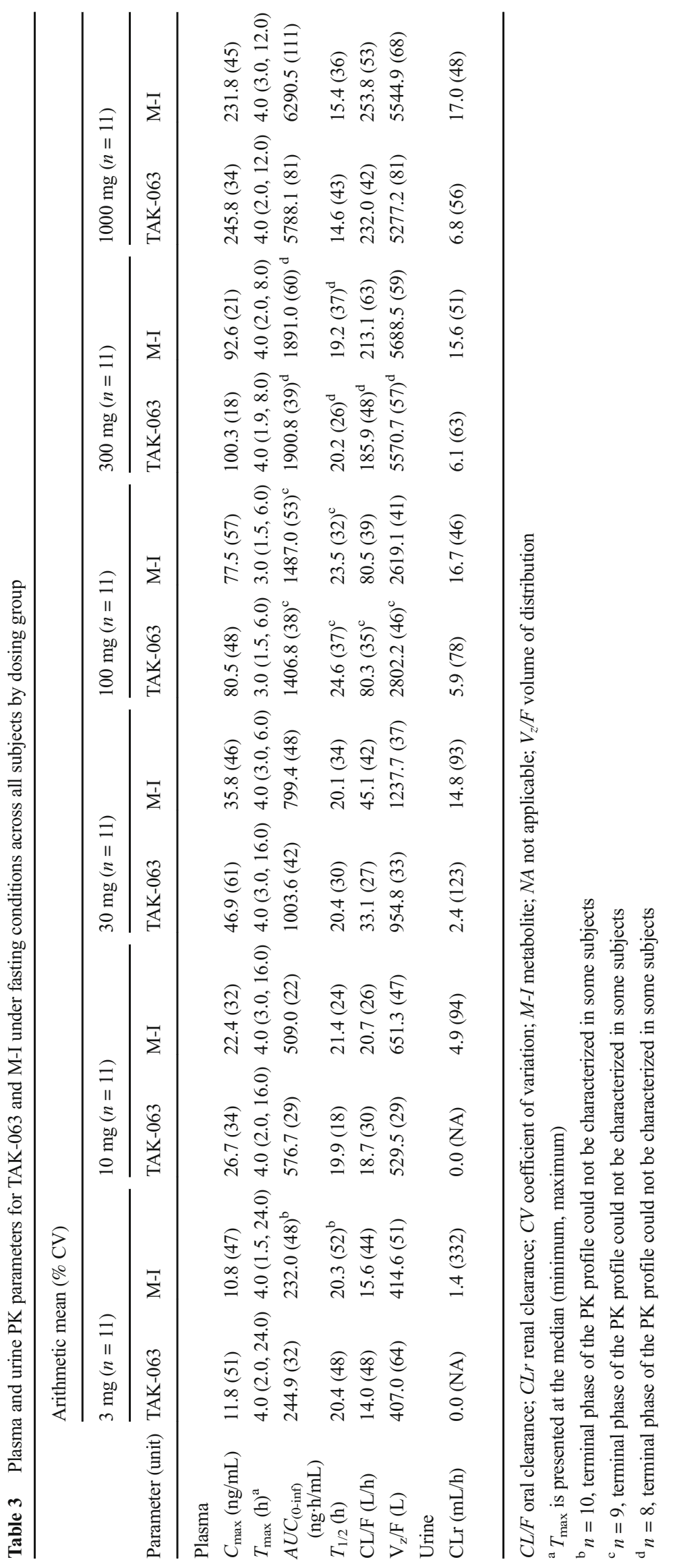




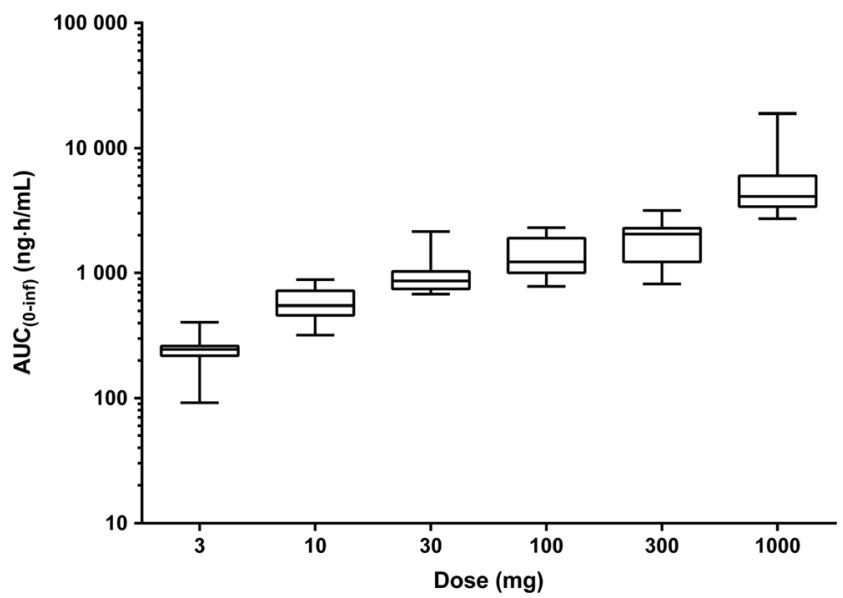

Fig. 2 Box plots ${ }^{\mathrm{a}}$ of TAK-063 exposure under fasting conditions across all subjects by dosing group. ${ }^{\mathrm{a}}$ Middle lines of boxes represent median values; top and bottom borders show lower and upper quartiles; whiskers represent minima and maxima

processing speed, psychomotor speed, and visual memory. In addition, statistically significant effects were noted at the 2-h timepoint in composite memory, reaction time, and verbal memory; the effects in these domains were not significant at the 6-h timepoint. A decrease from baseline in domain score in these cognitive domains, indicative of impairment, was generally observed following TAK-063 administration relative to the changes observed after placebo administration (Online Resource 6). No statistically significant differences were observed between Japanese and non-Japanese subjects for any of the neurocognitive domains tested (data not shown).

\section{Discussion}

In this single-ascending-dose study, the PK, PD, safety, and tolerability profiles of TAK-063 in healthy subjects were evaluated under fasting and fed conditions. The results show that TAK-063 is safe and well tolerated following administration of a single dose in Japanese and non-Japanese volunteers. Specifically, treatment with a single dose of TAK-063 was not associated with hyperprolactinemia, hyperglycemia, or

Table 4 PK parameters for food-effect arm following oral administration of $100 \mathrm{mg}$ TAK-063 in Japanese and non-Japanese subjects

\begin{tabular}{|c|c|c|c|c|}
\hline & \multicolumn{2}{|l|}{ TAK-063 } & \multicolumn{2}{|l|}{ M-I } \\
\hline & Estimate & $90 \% \mathrm{CI}$ & Estimate & $90 \% \mathrm{CI}$ \\
\hline$C_{\max }(\mathrm{ng} / \mathrm{mL})$ & 1.86 & $1.54,2.25$ & 1.95 & $1.63,2.33$ \\
\hline$A U C_{(0-\mathrm{inf})}(\mathrm{ng} \cdot \mathrm{h} / \mathrm{mL})$ & 1.78 & $1.52,2.09$ & 1.75 & $1.44,2.12$ \\
\hline
\end{tabular}

$C I$ confidence interval; $M-I$ metabolite other metabolic disturbances that are often observed with current antipsychotics (Henderson et al. 2005; Lieberman et al. 2005; Miyamoto et al. 2005). Somnolence was the most common TEAE associated with TAK-063 treatment. Across all doses, the incidence of EPS-like AEs was negligible, occurring in only one Japanese patient at $30 \mathrm{mg}$ (mild muscle tightness); it resolved without intervention. This may suggest that single doses of TAK-063 may have a relatively low propensity for EPS. No adverse events were considered dose-limiting, and a maximum tolerated dose was not defined.

TAK-063 and M-I exposure increased in a dosedependent manner. The nonlinear PK of TAK-063 may be due to limitations in drug absorption and oral bioavailability; the low aqueous solubility of TAK-063 is consistent with preclinical toxicology studies in which the drug was excreted in the feces, suggesting that the test article was not absorbed. Drug absorption and oral bioavailability can be increased through coadministration with food, and TAK-063 treatment under fed conditions exhibited slower absorption and increased oral bioavailability. These results are consistent with previous studies in animal models assessing TAK-063's solubility in the fed state.

The analyses of cognition data suggest that single doses of TAK-063 $\geq 100 \mathrm{mg}$ cause impairments in cognitive flexibility, executive functioning, processing and psychomotor speed, and visual memory. While the small sample size limits the interpretation of the cognition results, higher incidences and severity of somnolence were also reported at higher doses of TAK-063 and these effects may be similar to the effects on cognition of other sedative or hypnotic agents (Miyata et al. 2015; Stranks and Crowe 2014). Together, these data will inform subsequent analyses of TAK-063 studies in subjects with schizophrenia.

Selective PDE10A inhibitors represent a potentially new strategy in the treatment of schizophrenia, with preliminary evidence supporting a favorable safety and tolerability profile, and preclinical studies suggest PDE-10A inhibitors may have the potential for efficacy across multiple symptom domains (Grauer et al. 2009; Kehler and Nielsen 2011; Suzuki et al. 2015). The data reported in this study demonstrate the safety and tolerability of TAK063 following a single dose of up to $1000 \mathrm{mg}$ and support once-daily dosing. Taken together, these preliminary findings are encouraging and support the need for additional studies of TAK-063 in patients with schizophrenia. A clinical trial to characterize the PK and PD profiles of multiple rising doses of TAK-063 in patients with schizophrenia has recently been completed (NCT01879722). A phase 2 study of the efficacy and safety of TAK-063 for the treatment of acute exacerbations of the symptoms of schizophrenia is currently being conducted (NCT02477020). 
Acknowledgments Some data contained in this manuscript were presented in abstract form (Tsai M, Macek T, Chrones L, Xie J, Gevorkyan H. Safety, Tolerability and Pharmacokinetics of TAK063, a PDE10A Inhibitor, After a Single Dose in Healthy Japanese and Non-Japanese Subjects. American Society for Clinical Psychopharmacology 2015).

Contributors Max Tsai, Thomas Macek, and Lambros Chrones designed the study or were primarily responsible for the sponsor oversight and conduct of the study. Max Tsai did the PK analyses while Jinhui Xie did the statistical analysis. Hakop Gevorkyan was the principal investigator. All authors contributed to and have approved the final manuscript. Editorial support was provided by inVentiv Medical Communications, New York, NY. inVentiv prepared the preliminary draft of the manuscript under the direction of the lead author, Max Tsai, PhD. Dr. Tsai and the other authors maintained full editorial control of the manuscript and its conclusions throughout its development.

\section{Compliance with ethical standards}

Role of funding source Funding for this study was provided by Takeda Pharmaceutical Company, Limited.

Conflicts of interest Max Tsai, Thomas Macek, Lambros Chrones, and Jinhui Xie are employees of Takeda Development Center Americas, Inc., Deerfield, IL. Hakop Gevorkyan is an employee of California Clinical Trials Medical Group, Glendale, CA, and was Principal Investigator of the study.

Open Access This article is distributed under the terms of the Creative Commons Attribution 4.0 International License (http:// creativecommons.org/licenses/by/4.0/), which permits unrestricted use, distribution, and reproduction in any medium, provided you give appropriate credit to the original author(s) and the source, provide a link to the Creative Commons license, and indicate if changes were made.

\section{References}

Alexander GE, DeLong MR, Strick PL (1986) Parallel organization of functionally segregated circuits linking basal ganglia and cortex. Annu Rev Neurosci 9:357-381

Citrome L (2014) Unmet needs in the treatment of schizophrenia: new targets to help different symptom domains. J Clin Psychiatry 75(Suppl 1):21-26

de Araujo AN, de Sena EP, de Oliveira IR, Juruena MF (2012) Antipsychotic agents: efficacy and safety in schizophrenia. Drug Healthc Patient Saf 4:173-180

Farde L, Nordstrom AL, Wiesel FA, Pauli S, Halldin C, Sedvall G (1992) Positron emission tomographic analysis of central D1 and D2 dopamine receptor occupancy in patients treated with classical neuroleptics and clozapine. Relation to extrapyramidal side effects. Arch Gen Psychiatry 49(7):538-544

Fujishige K, Kotera J, Omori K (1999) Striatum- and testis-specific phosphodiesterase PDE10A isolation and characterization of a rat PDE10A. Eur J Biochem 266(3):1118-1127

Fukunaga S, Kusama M, Arnold FL, Ono S (2011) Ethnic differences in pharmacokinetics in new drug applications and approved doses in Japan. J Clin Pharmacol 51:1237-1240

Ginovart N, Kapur S (2012) Role of dopamine D(2) receptors for antipsychotic activity. Handb Exp Pharmacol 212:27-52
Grauer SM, Pulito VL, Navarra RL, Kelly MP, Kelley C, Graf R, Langen B, Logue S, Brennan J, Jiang L, Charych E, Egerland U, Liu F, Marquis KL, Malamas M, Hage T, Comery TA, Brandon NJ (2009) Phosphodiesterase 10A inhibitor activity in preclinical models of the positive, cognitive, and negative symptoms of schizophrenia. J Pharmacol Exp Ther 331(2):574-590

Gualtieri CT, Johnson LG (2006) Reliability and validity of a computerized neurocognitive test battery, CNS vital signs. Arch Clin Neuropsychol 21:623-643

Heckman PR, van Duinen MA, Bollen EP, Nishi A, Wennogle LP, Blokland A, Prickaerts J (2016) Phosphodiesterase inhibition and regulation of dopaminergic frontal and striatal functioning: clinical implications. Int J Neuropsychopharmacol. doi:10.1093 /ijnp/pyw030

Henderson DC, Cagliero E, Copeland PM, Borba CP, Evins E, Hayden D, Weber MT, Anderson EJ, Allison DB, Daley TB, Schoenfeld D, Goff DC (2005) Glucose metabolism in patients with schizophrenia treated with atypical antipsychotic agents: a frequently sampled intravenous glucose tolerance test and minimal model analysis. Arch Gen Psychiatry 62(1):19-28

Kehler J, Nielsen J (2011) PDE10A inhibitors: novel therapeutic drugs for schizophrenia. Curr Pharm Des 17(2):137-150

Kreitzer AC (2009) Physiology and pharmacology of striatal neurons. Annu Rev Neurosci 32:127-147

Lehman AF, Lieberman JA, Dixon LB, McGlashan TH, Miller AL, Perkins DO, Kreyenbuhl J, American Psychiatric Association; Steering Committee on Practice Guidelines (2004) Practice guideline for the treatment of patients with schizophrenia, second edition. Am J Psychiatry 161(2 Suppl):1-56

Leucht S, Corves C, Arbter D, Engel RR, Li C, Davis JM (2009) Secondgeneration versus first-generation antipsychotic drugs for schizophrenia: a meta-analysis. Lancet 373(9657):31-41

Lieberman JA, Stroup TS, McEvoy JP, Swartz MS, Rosenheck RA, Perkins DO, Keefe RS, Davis SM, Davis CE, Lebowitz BD, Severe J, Hsiao JK, Clinical Antipsychotic Trials of Intervention Effectiveness (CATIE) Investigators (2005) Effectiveness of antipsychotic drugs in patients with chronic schizophrenia. $\mathrm{N}$ Engl $\mathrm{J}$ Med 353(12):1209-1223

Macpherson T, Morita M, Hikida T (2014) Striatal direct and indirect pathways control decision-making behavior. Front Psychol 5:1301

Miyamoto S, Duncan GE, Marx CE, Lieberman JA (2005) Treatments for schizophrenia: a critical review of pharmacology and mechanisms of action of antipsychotic drugs. Mol Psychiatry 10(1):79-104

Miyata A, Iwamoto K, Kawano N, Kohmura K, Yamamoto M, Aleksic B, Ebe K, Noda A, Noda Y, Iritani S, Ozaki N (2015) The effects of acute treatment with ramelteon, triazolam, and placebo on driving performance, cognitive function, and equilibrium function in healthy volunteers. Psychopharmacology (Berl) 232(12):21272137

Newcomer JW (2007) Metabolic considerations in the use of antipsychotic medications: a review of recent evidence. J Clin Psychiatry 68(Suppl 1):20-27

Perez-Costas E, Melendez-Ferro M, Roberts RC (2010) Basal ganglia pathology in schizophrenia: dopamine connections and anomalies. J Neurochem 113(2):287-302

Sano H, Nagai Y, Miyakawa T, Shigemoto R, Yokoi M (2008) Increased social interaction in mice deficient of the striatal medium spiny neuron-specific phosphodiesterase 10A2. J Neurochem 105(2):546-556

Seeger TF, Bartlett B, Coskran TM, Culp JS, James LC, Krull DL, Lanfear J, Ryan AM, Schmidt CJ, Strick CA, Varghese AH, Williams RD, Wylie PG, Menniti FS (2003) Immunohistochemical localization of PDE10A in the rat brain. Brain Res 985(2):113-126 
Siuciak JA, McCarthy SA, Chapin DS, Fujiwara RA, James LC, Williams RD, Stock JL, McNeish JD, Strick CA, Menniti FS, Schmidt CJ (2006) Genetic deletion of the striatum-enriched phosphodiesterase PDE10A: evidence for altered striatal function. Neuropharmacology 51(2):374-385

Song XJ, Wang ZB, Gan Q, Walters ET (2006) cAMP and cGMP contribute to sensory neuron hyperexcitability and hyperalgesia in rats with dorsal root ganglia compression. J Neurophysiol 95(1):479-492

Stranks EK, Crowe SF (2014) The acute cognitive effects of zopiclone, zolpidem, zaleplon, and eszopiclone: a systematic review and metaanalysis. J Clin Exp Neuropsychol 36:691-700
Surmeier DJ, Ding J, Day M, Wang Z, Shen W (2007) D1 and D2 dopamine-receptor modulation of striatal glutamatergic signaling in striatal medium spiny neurons. Trends Neurosci 30(5):228-235

Suzuki K, Harada A, Shiraishi E, Kimura H (2015) In vivo pharmacological characterization of TAK-063, a potent and selective phosphodiesterase $10 \mathrm{~A}$ inhibitor with antipsychotic-like activity in rodents. J Pharmacol Exp Ther 352(3):471-479

Tarrier N (1987) An investigation of residual psychotic symptoms in discharged schizophrenic patients. Br J Clin Psychol 26(Pt 2):141-143

Threlfell S, Sammut S, Menniti FS, Schmidt CJ, West AR (2009) Inhibition of phosphodiesterase $10 \mathrm{~A}$ increases the responsiveness of striatal projection neurons to cortical stimulation. J Pharmacol Exp Ther 328(3):785-795 